Int. J. Dev. Biol. 59: 435-442 (2015)

doi: $10.1387 / \mathrm{ijdb} .150310 \mathrm{ht}$

\title{
Ras-Related Nuclear Protein is required for late developmental stages of retinal cells in zebrafish eyes
}

\author{
CHENG-YUNG LIN", ${ }^{\# \text {, HING-YEN HUANG }}{ }^{\#, 2}$, PO-NIEN LU\#,2, CHIEN-WEI LIN², KUANG-MING LU² \\ and HUAI-JEN TSAl ${ }^{1, *}$ \\ ${ }^{1}$ Institute of Biomedical Sciences, Mackay Medical College, New Taipei City and \\ ${ }^{2}$ Institute of Molecular and Cellular Biology, National Taiwan University, Taipei, Taiwan
}

\begin{abstract}
Ras-related nuclear protein (Ran) is involved in cell division by regulating nucleocytoplasmic transport and modulating the assembly of tubulin. However, its function in embryonic development is unclear. We used zebrafish to study the roles of Ran in eye development. The ran transcripts were restrictedly expressed in head and eyes after the pharyngula stage. The microphthalmos, in which no ordered layers with differentiated retinal cells were detected, was observed in the ran-deficient embryos. They exhibited faster decline cyclinD1-expressed cells, suggesting that cell cycle regulation in retinae was defective. The apoptotic signals in the retinae of ran-deficient embryos remained low at early ( $24 \mathrm{hpf}$ ) stage. Early eye field specification markers, $r \times 1$ and pax6, were only slightly affected, and markers for establishing axon migration, fgf8 and pax2, were normally expressed, suggesting Ran is not required in the early stages of eye development. However, the early optic nerve differentiation marker p57kip2 was not expressed at middle (48 hpf) and late (72 hpf) stages. We also observed a decrease in the retinal neuron proteins $\mathrm{HuC}$ and Neurolin. The proneural gene ath5, which first determines the cell fate of the developing ganglion cell layer, was undetectable. Furthermore, we found that Ran was associated with ADP-ribosylation factor-like protein 6-interacting protein 1 (Arl6ip1), which plays a role in retinal development, suggesting that Ran associates with Arl6ip1 to regulate retinal development. Therefore, while the effects of Ran are minimal during early specification of the eye field, Ran is required for proliferation and differentiation of retinal cells at later developmental stages.
\end{abstract}

KEY WORDS: Ran, zebrafish, retinal cells, eye development

\section{Introduction}

Ras-related nuclear protein (Ran) is a small GTP-binding protein and member of the Ras superfamily. Since Ran lacks a membrane domain and is mostly detected in the nucleus, it is generally not considered as a typical signal transduction molecule (Sazer, 1996). Originally, Ran was discovered to play roles in nucleocytoplasmic transport in both statically and actively proliferating cells. By regulating the assembling and disassembling of the Ran-binding transportation proteins and their cargos, Ran is able to regulate transportation entering and leaving the nucleus (Gorlich \& Kutay, 1999). Thus, while originally known to be a master regulator of nucleocytoplasmic transport (Melchior et al., 1995), Ran is also required in spindle formation and nuclear envelope assembly during mitosis (Hetzer et al., 2002), and it has been described as a central element of a conserved signaling network, playing crucial roles in mitotic regulation (Ciciarello et al., 2007). Importantly, further studies indicate that the roles Ran plays in mitosis are likely independent from the nucleocytoplasmic transportation mechanism. For example, evidences indicate that Ran promotes tubulin polymerization and, consequently, stabilizes the microtubule structure (Dasso, 2001; Sazer \& Dasso, 2000). Additionally, Ran and its binding protein RanBPM have been reported to play roles in signal transduction and affect the differentiation of neurons (Cheng et al., 2005; Togashi et al., 2006). Recently, the overexpression of

Abbreviations used in this paper: Arl6ip, ADP-ribosylation factor-like protein 6-interacting protein; RAN, Ras-related nuclear protein.

\footnotetext{
*Address correspondence to: Huai-JenTsai. No. 46, Sec. 3, Zhong-Zheng Rd., Sanzhi Dist., NewTaipei City, Taiwan 25245. Tel: 886-2-2636-0303 ext. 1724. E-mail: hjtsai@mmc.edu.tw
}

\#Note: The indicated authors contributed equally to this paper.

Supplementary Material (three figures) for this paper is available at: http://dx.doi.org/10.1387/ijdb.150310ht

Accepted: 19 November 2015.

ISSN: Online 1696-3547, Print 0214-6282 


\section{ran mRNA}

A
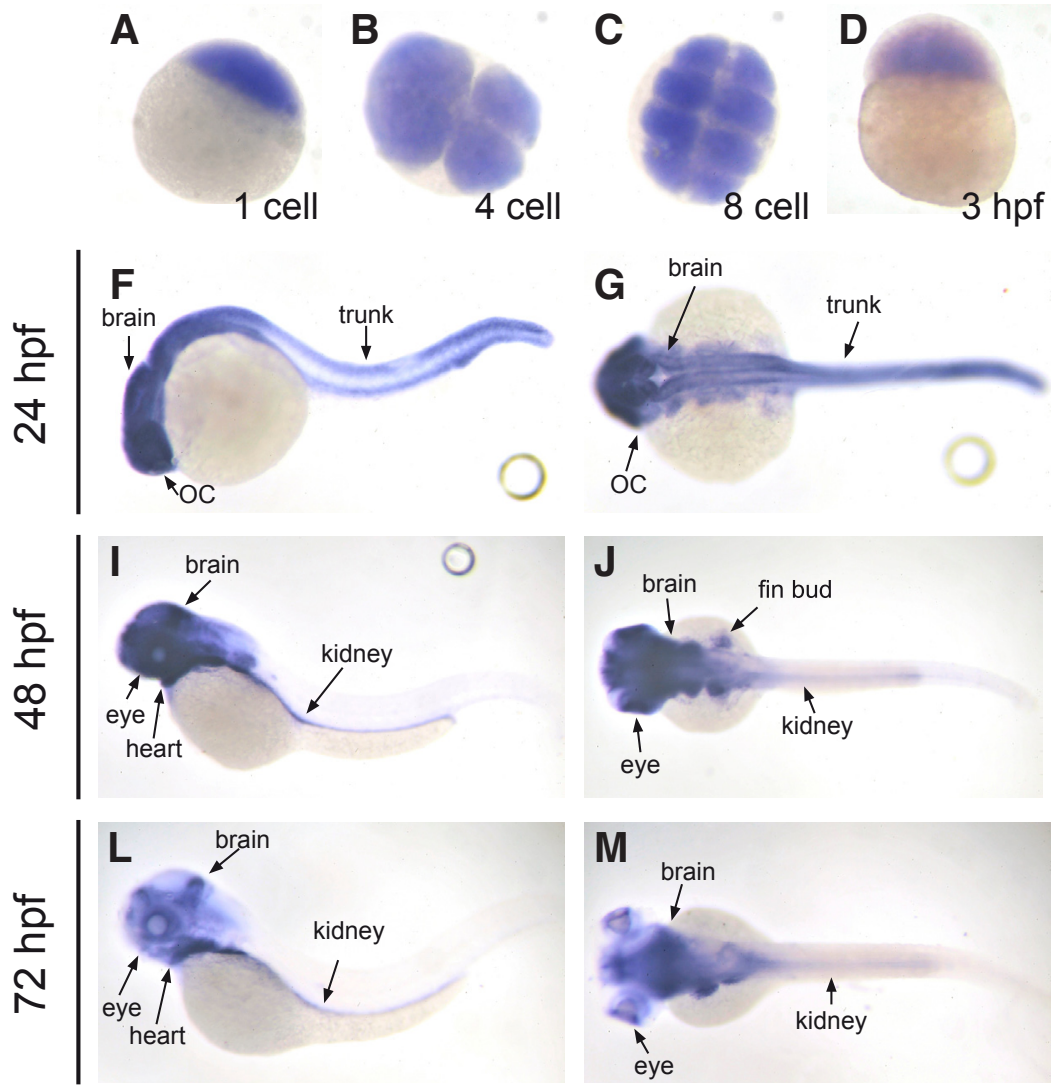
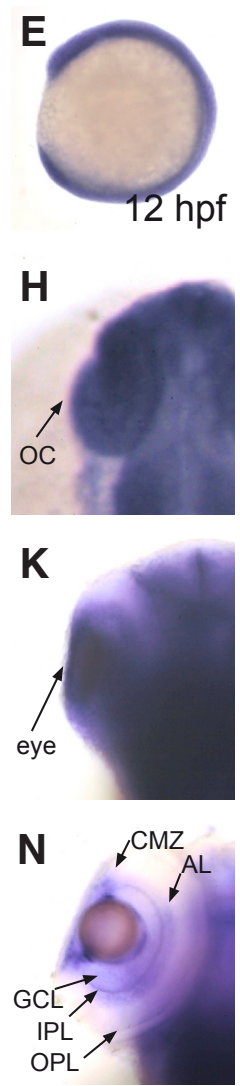

Fig. 1. The spatiotemporal expression pattern of ran mRNA in developing zebrafish embryo. (A-E) From onecell stage up to $12 \mathrm{hpf}$, ran mRNA was detected on the whole embryo. (A,D,E) Lateral view; $(\mathbf{B}, \mathbf{C})$ animal polar view. (FH) At 24 hpf, while ran mRNA can still be detected in brain and optic cup, the level of ran mRNA started to decrease at the trunk area. (I-K) At $48 \mathrm{hpf}$, ran mRNA was highly expressed in brain, eyes, heart, fin buds and kidney, but not in trunk. (L-N) At 72 hpf, ran mRNA was expressed in brain, heart and kidney. In the eye, ran mRNA was detected on $C M Z, G C L$, IPL, $A L$ and OPL. $(\mathbf{F}, \mathbf{I}, \mathbf{L})$ Lateral view with anterior to the left; $(\mathbf{G}, \mathbf{J}, \mathbf{M})$ dorsal view with anterior to the left; $(\mathbf{H}, \mathbf{K}, \mathbf{N})$ dorsal view with anterior to the top, focused on the optic cup area, and only the left eyes are presented to show better detail. $A L$, amacrine layer; $C M Z$, ciliary marginal zone; GCL, ganglion cell layer; IPL, inner plexiform layer; OC, optic cup; OPL, outer plexiform layer. All images are representative of at least three repeats with $n>25$ for each repeat.
Ran has also been linked to cancer progression (Deng et al., 2014).

Ran is highly conserved among vertebrates; for example, zebrafish Ran exhibits $94.9 \%$ similarity to human Ran (Liao et al., 1997). As revealed in previous RT-PCR results, ran mRNA exists in brain, eye, gill, heart, liver, intestine, ovary, skin and muscle of adult zebrafish (Liao et al., 1997). While it has been reported that in the mouse model of frontotemporal lobar degeneration, retinal thinning phenotype has been reported as associated with altered Ran expression level (Ward et al., 2014), the biological function of Ran in embryonic development is largely unclear. In this study, we used zebrafish as an animal model in developmental biology to study the roles of Ran in eye development. We first utilized whole-

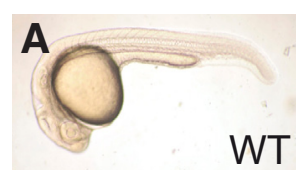

B
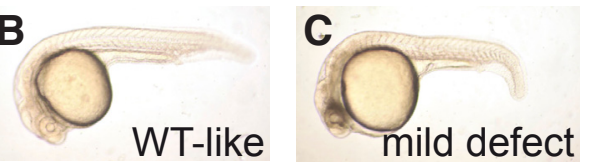

$E$
0
0
0
0
0
0
$\frac{1}{0}$
40
0
0
$\frac{\pi}{0}$
0
0
0
0

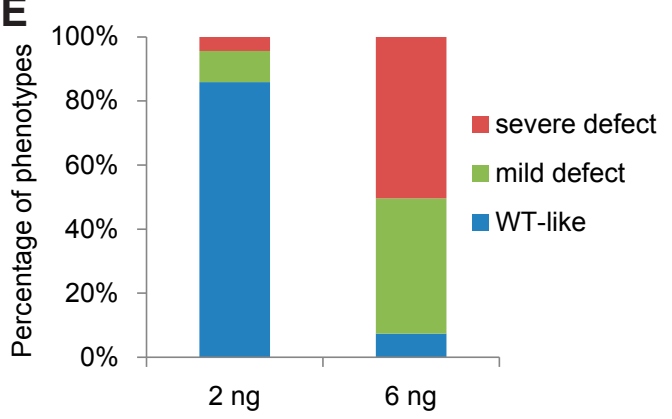

amont of ran-MO1 injected

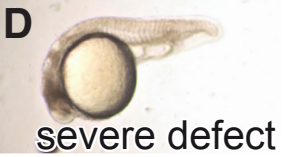

\section{severe defect}
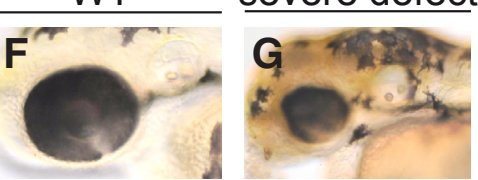

옴

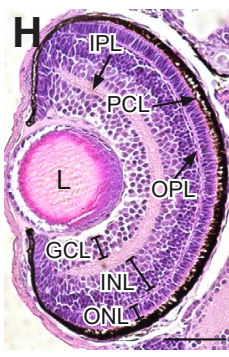

Fig. 2. The phenotypes of Ran deficiency (A-D) Typical phenotypes of live WT and randeficient embryos at $24 \mathrm{hpf}$. (E) The distribution of phenotypes when 2 ng and 6 ng of ran-MO1 were injected. The injection experiment was repeated three times with pooled $n=411$ and 627 for 2 ng and 6 ng injection groups, respectively. (F-G) Typical images of live WT eye and the eye with severe ran-deficient phenotype at $48 \mathrm{hpf}$. All live embryo images are lateral view with anterior to the left. (H-I) H\&E staining of WT eye and the eye with severe ran-deficient phenotype at $72 \mathrm{hpf}$. All H\&E staining images are coronal sections with anterior to the top. $G C L$, ganglion cell layer; INL, inner nuclear layer; IPL, inner plexiform layer; ONL, outer nuclear layer; $O P L$, outer plexiform layer; $P C L$, pigment cell layer. Scale bars, $50 \mu \mathrm{m}$. All images of Ran-deficient embryos were injected with 6 ng of ran-MO1. All images are representative of at least three repeats with pooled $n>400$. 
mount in situ hybridization (WISH) to demonstrate the existence of ran transcript in the developing eye. To further study the roles of Ran in retinal development, we used loss-of-function strategy to specifically knock down Ran level in zebrafish embryos, followed by examining phenotypes caused by Ran deficiency. Finally, we verified the binding ability of Ran and ADP-ribosylation factor-like 6 interacting protein 1 (Arl6ip1), a protein that has been known to play an important role in retinal development of zebrafish embryos (Huang et al., 2009), concluding that Ran associates with Arl6ip1 to play a role in the development of zebrafish retina.

\section{Results}

\section{The spatiotemporal expression pattern of ran in the developing zebrafish embryo}

To first understand the expression pattern of ran at different stages of zebrafish development, especially in the eye, we conducted WISH on zebrafish embryos at one-cell, two-cell, four-cell, eight-cell, 3 hpf, 12 hpf, 24 hpf, 48 hpf and 72 hpf stages. From the one-cell stage to the $12 \mathrm{hpf}$ stage, we found that ran mRNA was expressed in the whole embryo (Fig. $1 \mathrm{~A}-\mathrm{E}$ ). At $24 \mathrm{hpf}$, the ran signal started to decrease in the trunk area, while remaining strongly expressed in brain and optic cup (Fig. 1 F-H). At 48 hpf, the ran signal was nearly undetectable in the trunk area, but strongly expressed in brain, eye, heart, fin bud and kidney (Fig. 1 I-K). The expression pattern of ran at $72 \mathrm{hpf}$ was similar to the pattern at $48 \mathrm{hpf}$, although the overall signal strength was mildly decreased (Fig. $1 \mathrm{~L}-\mathrm{N}$ ). Notably, in the 72-hpf eye, ran signal was detected in the ciliary marginal zone (CMZ), ganglion cell layer (GCL), inner plexiform layer (IPL) and amacrine cell layer (AL), but not in the outer nuclear layer (ONL).

\section{Ran deficiency led to microphthalmos and disrupted retinal structures}

To further investigate the role ran plays in zebrafish embryonic development, we designed two MOs' sequences that specifically cause the loss the Ran function. Ran-MO1 targeted the sequence including the translation start site, while ran-MO2 targeted a section of the 5'UTR of ran mRNA. In the embryos injected with 2 to $6 \mathrm{ng}$ of ran-MO1, we found that embryos exhibited significant phenotypes, including smaller eyes (microphthalmos), deformed heads and bent or shorter tails (Fig. 2 A-D). We classified the embryos with smaller eyes and slightly bent tails as having mild phenotype, while embryos with smaller eyes, deformed heads and short tails were classified as having severe phenotype. When $6 \mathrm{ng}$ of ran-MO1 were injected, $50 \%$ of the embryos exhibited severe phenotype, $42 \%$ of the embryos exhibited mild phenotype, and only $7 \%$ of the embryos were wild type (WT)-like (Fig. 2E). On the contrary, when 2 ng of ran-MO1 were injected, only $5 \%$ of the embryos exhibited severe phenotype, and $10 \%$ of the embryos exhibited mild phenotype, indicating dose-dependent effects (Fig. $2 \mathrm{E})$. To verify the effectiveness of the $\mathrm{MO}$, we used Western blot analysis to confirm that ran-MO1 specifically suppressed the level of Ran protein (Supplementary Fig. 1). To demonstrate that the phenotypes we observed were specific, ran-MO2 targeting a different part of ran mRNA was injected, and similar dose-dependent phenotypes were observed (Supplementary Fig. 2).

Since the microphthalmos phenotype became more severe at 48 hpf, we conducted tissue sectioning and Hematoxylin-Eosin staining to further identify the eye-related phenotype of ran-deficient embryos. At 48 hpf, we observed that WT eyes had already developed a six-layered structure with distinctly shaped cells located in each layer (Fig. 2F). However, in the eyes of ran-knockdown embryos, all cells were similarly shaped, and no layers could be identified, except for the pigment cell layer (PCL), resulting from the deep color (Fig. 2G).

\section{Ran deficiency had only minor effects on early eye differen- tiation}

To further understand the roles of Ran on eye development, we first examined the effects of Ran deficiency on the expression of two eye field transcription factors (EFTFs), rx1 and pax6, which have been demonstrated to be required for the early specification
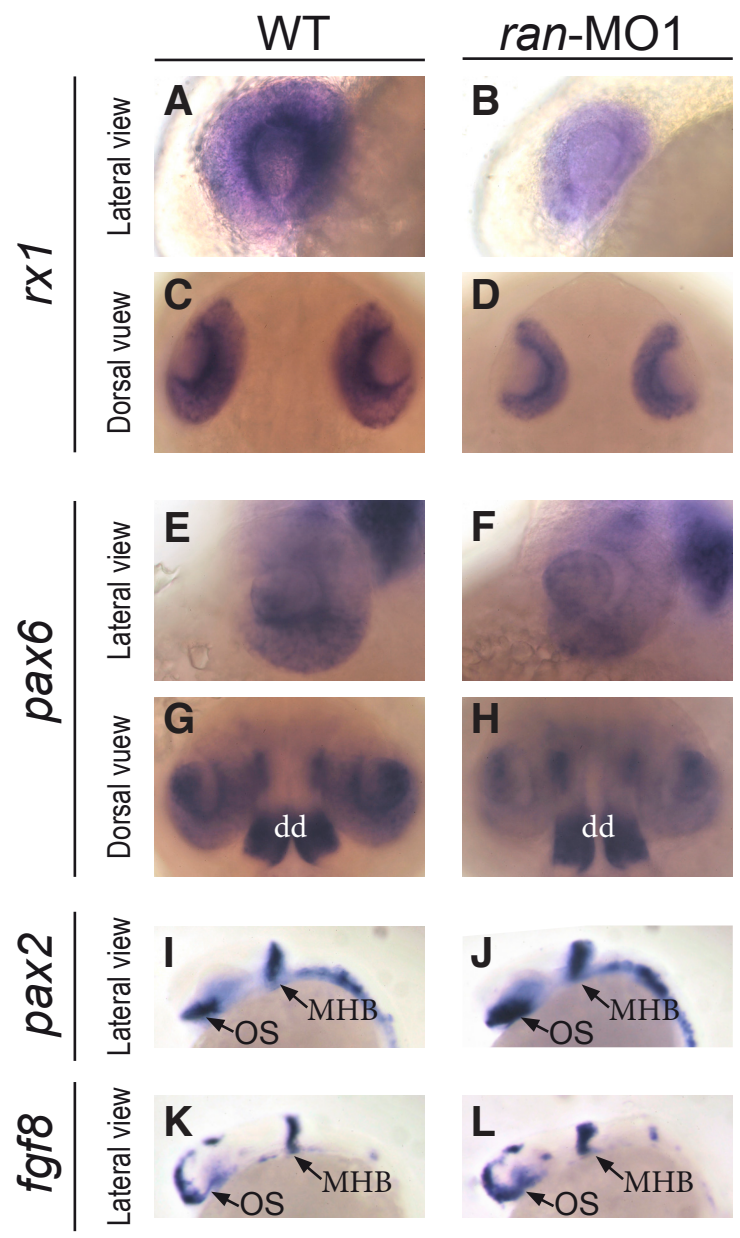

Fig. 3. The effects of Ran deficiency on the expression of early eye differentiation markers. (A-D) Typical expression patterns of rx1 mRNA on the eyes of WT and Ran-deficient embryos. While sizes are different, note that the patterns of pax 6 were not significantly changed. (E-H) Typical expression patterns of pax6 mRNA on the eyes of WT and ran-deficient embryos. dd: dorsal diencephalon. $(\mathbf{I}, \mathbf{J})$ The expression patterns of pax2 mRNA on the eyes of WT and ran-deficient embryos. (K,L) The expression patterns of fgf8 mRNA on the eyes of WT and Ran-deficient embryos. (A, $\mathbf{B}, \mathbf{E}, \mathbf{F}, \mathbf{I}-\mathbf{L})$ Lateral view with anterior to the left; $(\mathbf{C}, \mathbf{D}, \mathbf{G}, \mathbf{H})$ dorsal view with anterior to the top. MHB, midbrain/hindbrain boundary; OS, optic stalk. All images are representative of at least three repeats with $n>25$ for each repeat. 

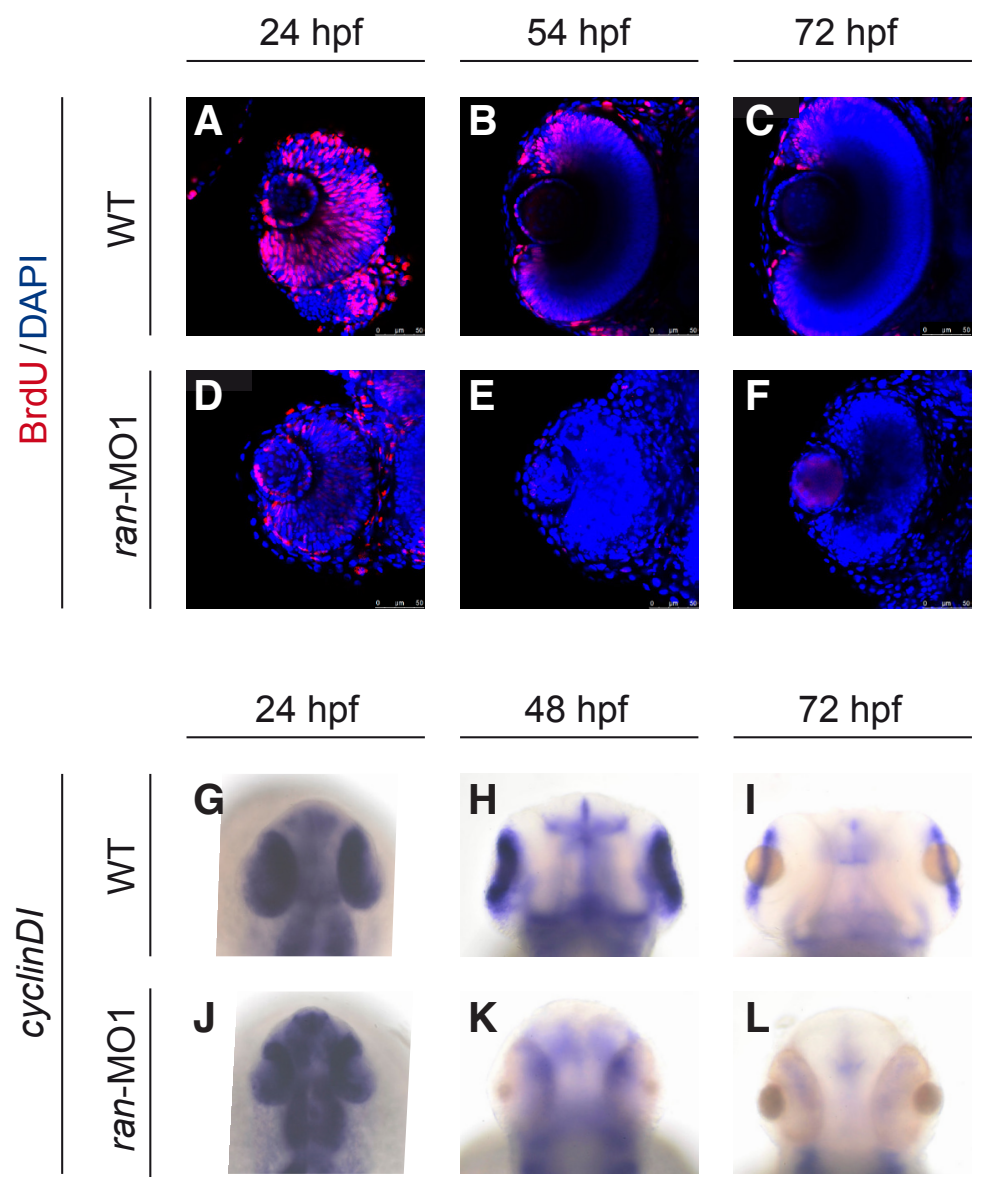

Fig. 4. Ran deficiency impaired eye cell proliferation. (A-F) Temporal dynamics of cell proliferation on the eyes of WT and ran-deficient embryos. All images are coronal sections with anterior to the top. Red, BrdU; Blue, DAPI. Scale bars indicate $50 \mu \mathrm{m}$. (G-L) Temporal dynamics of cell proliferation marker cyclinD1 mRNA on the eyes of WT and ran-deficient embryos. All images are dorsal view with anterior to the top. All images are representative of at least three repeats with $n>25$ for each repeat.

of vertebrate eye (Zaghloul \& Moody, 2007). Between WT and ran-deficient embryos, we found that the expression patterns of $r \times 1$ and pax6 were similar, although the expression level of both genes was slightly lower in the ran-deficient embryos (Fig $3 \mathrm{~A}-\mathrm{H}$ ).

We then examined the effects of Ran deficiency on the optic stalk, a structure that provides a pathway for axon migration from the retina. We used fgf8 and pax 2 as optic stalk gene markers (Heisenberg et al., 1999; Krauss et al., 1991) and found that the ran-deficient embryos exhibited no significant changes in expression pattern or expression level of either fgf8 or pax2 (Fig. $3 \mathrm{I}-\mathrm{L}$ ).

\section{Ran deficiency led to reduced cell proliferation in eye}

Since the early specification of eye-related structures was not significantly disrupted by the loss of Ran function, we further investigated the roles of Ran in later eye development. Using BrdU labeling, we found that the ran-knockdown zebrafish exhibited significantly reduced cell proliferation during the later stages of eye development (Fig. 4 A-F). At both 24 hpf and 72 hpf, TUNEL assay also revealed that apoptosis remained at a low level in both WT and ran-deficient embryos (Supplementary Fig. 3).
We also examined the expression of cyclinD1, a gene required for G1/S transition (Pines, 1995). At $24 \mathrm{hpf}$, we found no difference between WT and ran-deficient embryos (Fig. 4G vs. 4J). However, at $48 \mathrm{hpf}$, while the expression level of cyclinD1 was reduced in WT eyes, it became nearly undetectable in the eyes of ran-deficient embryos (Fig. $4 \mathrm{H}$ vs. 4K). At $72 \mathrm{hpf}$, cyclinD1 was only expressed at the ciliary marginal zone (CMZ) of the WT eyes, while it was completely missing from the eyes of ran-deficient embryos (Fig. 4I vs. 4L).

\section{Ran is required for the differentiation of retinal neurons}

We then evaluated the role Ran plays in the differentiation of retina using several markers related to retinal differentiation, including p57kip2, deltaC, ath5, neuroD, HuC and DM-GRASP (Neurolin). The p57kip2 marker is a Cyclin-dependent kinase inhibitor known to inhibit CyclinD and consequently promotes differentiation in retina (Shkumatava \& Neumann, 2005). In WT eyes, we found that $p 57 k i p 2$ was moderately expressed at $24 \mathrm{hpf}$, highly expressed at $48 \mathrm{hpf}$, then reduced to a low level at 72 hpf. In ran-knockdown eyes, p57kip2 was also moderately expressed at $24 \mathrm{hpf}$, but gradually reduced at 48 and 72 hpf (Fig. 5 A-F).

We found that deltaC, a marker for retina progenitor cell (Smithers et al., 2000), was normally expressed in the eyes of ran-morphants at $24 \mathrm{hpf}$. However, at $48 \mathrm{hpf}$, while deltaC was normally decreasing in WT eyes, ran-defective embryos exhibited a more severely reduced deltaClevel. Furthermore, at $72 \mathrm{hpf}$, while deltaC was still detected in the CMZ of WT eyes, ran-defective eyes expressed no deltaC (Fig. 5 G-L).

Ath5 and NeuroD are both required for the determination of retinal fate (Kanekar et al., 1997; Ochocinska \& Hitchcock, 2009). While the expression level of ath5was highly dynamic in WT, it was variable in the ran-knockdown phenotype, reducing its levels significantly between 24 and $42 \mathrm{hpf}$ (Fig. 5 $\mathrm{M}-\mathrm{R}$ ). At $48 \mathrm{hpf}$, neuroD was expressed on the AL and ONL of WT eyes, while ran deficiency resulted in a reduced and unorganized neuroD expression pattern (Fig. 5S, 5T).

Finally, to verify the production of neurons in the retina, we examined the existence of HuC/D and Neurolin protein, markers for differentiated neurons (Kim et al., 1996). While both $\mathrm{HuC/D}$ and Neurolin were expressed on the GCL of WT eyes, both proteins were undetectable in the ran-deficient embryos (Fig. $5 \mathrm{U}-\mathrm{X}$ ), indicating that the differentiation of retinal neurons was completely blocked. Notably, since no deltaC expression was detected in the eyes of ran-knockdown fish at 48 hpf (Fig. 5K), the HuC- and Neurolin-negative cells were not likely to be progenitor cells. Thus, the defective retina observed in ran-deficient embryos more likely reflected completely failed differentiation rather than simply delayed development.

\section{Arl6ip1 is a binding partner of Ran}

In our previous study, we identified that ADP-ribosylation factorlike protein 6-interacting protein 1 (Arl6ip1, previously namedArl6ip) is required for the formation of retinal layers (Huang et al., 2009). Since Arl6ip1 deficiency and Ran deficiency are highly similar in the retinal phenotypes (Huang et al., 2009), we hypothesized that Ran and Arl6ip1 might be binding partners that function in the same signaling pathway. To verify our hypothesis, we conducted an Arl6ip1 immunoprecipitation assay on zebrafish total protein 
extract (Fig. 6A). Under Coomassie Brilliant Blue staining, we detected a protein signal that did not overlap with the molecular weight of Arl6ip or any other known background signal (Fig. 6A, lane 3 ). This protein band was then collected and submitted for LC-MS/MS analysis. Among the predicted sequences, Ran was identified as a candidate. We then conducted immunoprecipitation assay on both zebrafish total protein extracts and 293T whole cell lysates. In both cases, the Ran signal was detected in the Arl6ip1 immunoprecipitation, supporting the hypothesis that Ran is likely a binding partner of Arl6ip1 (Fig. 6B).

To further examine whether Arl6ip1 and Ran bind each other directly, we constructed Escherichia coli expression vectors that produced His-tagged Arl6ip1 (His-Arl6ip1) and Ran (His-Ran).
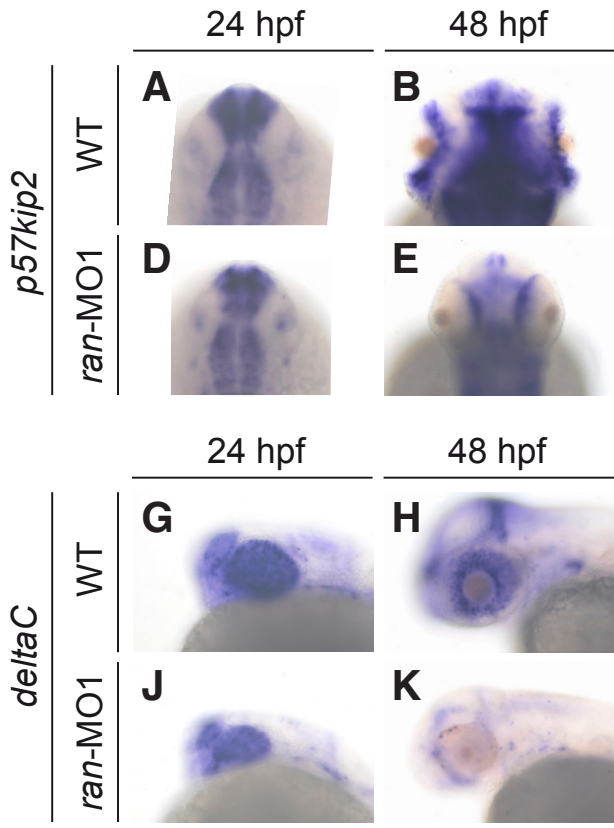

$48 \mathrm{hpf}$
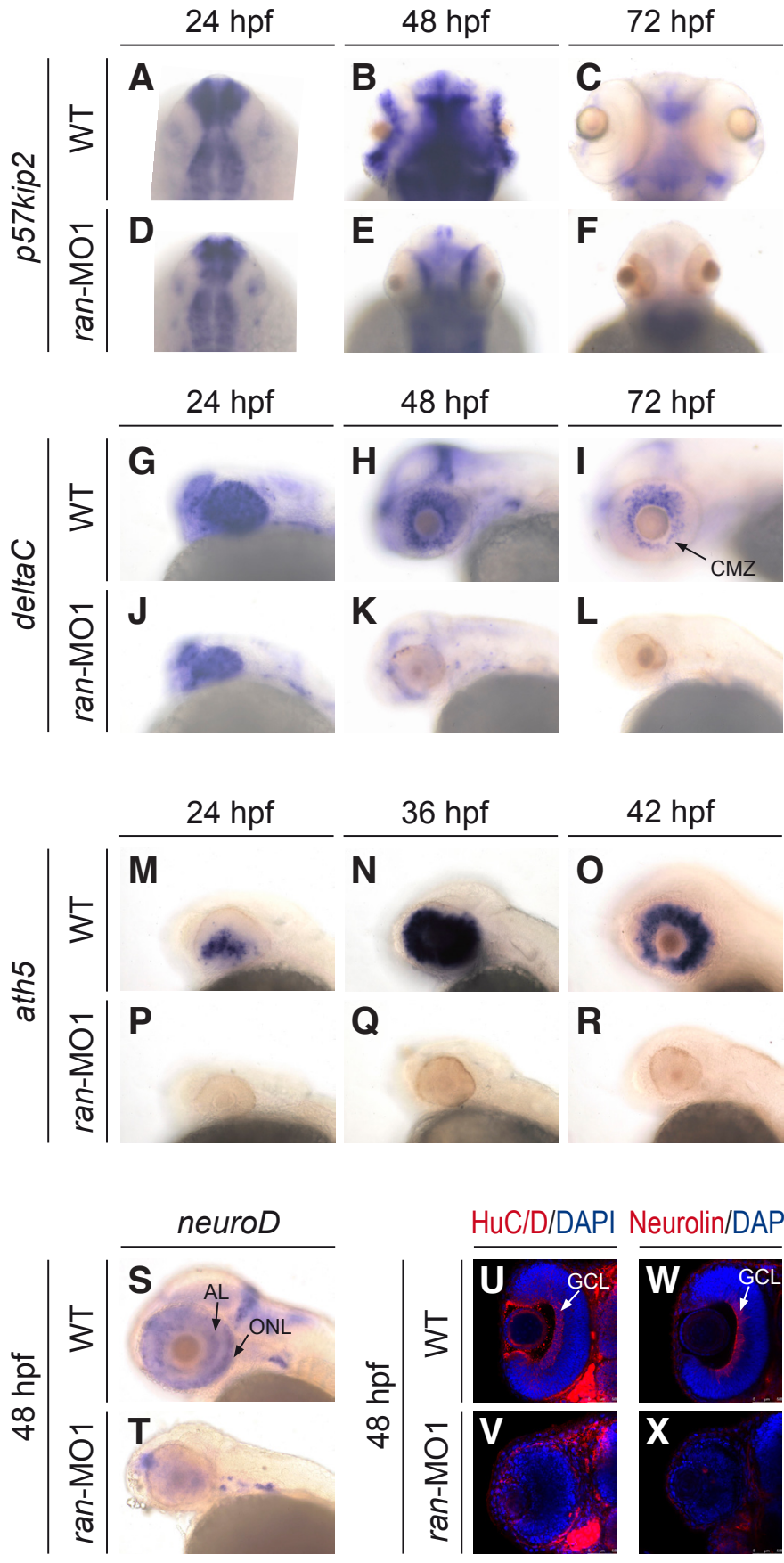

His-Arl6ip1 and His-Ran were then purified and subjected to in vitro immunoprecipitation assay. When the His-Arl6ip1 and HisRan mixture were pulled down by anti-Arl6ip1 antibody, we found that the Ran signal was detected in the precipitated fraction (Fig. $6 \mathrm{C})$. Such finding supported the hypothesis that the interaction between Arl6ip1 and Ran is direct.

\section{Discussion}

Ran plays minimal roles in early-stage eye development, but is critical at later stages of development

In this study, we investigated the roles of Ran in zebrafish eye development. While the effects of Ran deficiency were minimal during early gastrulation, we demonstrated that ran morphants exhibited smaller head, smaller eye, and bent trunk at later developmental stages. The development of brain and retina was severely defective, while the bent trunk phenotype was relatively minor. Such pattern also agreed with the spatiotemporal expression pattern of ran mRNAduring development, which gradually became restricted to the head area. Further studies were focused on the microphthalmos phenotype since it exhibited distinct defects in the establishment of retinal layers and optical nerve.

We first examined the effects of Ran deficiency on early specification of the eye field. Surprisingly, $r \times 1$, an early gene required for defining the area of optic primordium (Chuang \& Raymond, 2001), was not significantly affected. The genes defining future neural retinal and optic stalk, pax6 and pax2 (Chow et al., 2001), were also not significantly affected. Thus, we concluded that Ran functions at a later stage of eye development.

\section{Ran deficiency results in premature cell cycle exit and dif- ferentiation failure in retina}

To elucidate the etiology of microphthalmia, we employed TUNEL assay to detect whether the zebrafish retinae have any abnormal apoptotic signal at early and late stages. Since neither stage exhibited abnormal apoptosis, we then used BrdU to label and observe the retinae of ran-deficient embryos, showing that cell division was severely decreased in the eye. Especially at later stage, the BrdU signal was lost completely, indicating premature cell cycle exit.

The p57kip2 functions as a cyclin-dependent kinase inhibitor protein to induce differentiation through inhibiting cell cycle. Shkumatava and Neumann (2005) found that its loss of function resulted in a phenotype resembling sh mutant zebrafish. On the other hand, overexpression of $p 57$ kip 2 could rescue premature exit of cell cycle in shh mutant, resulting in precursor cell differentiation through inhibiting cyclinD1. We herein found that expression of

Fig. 5. Ran deficiency impaired eye cell differentiation. (A-R) Temporal dynamics of the expression of cell differentiation marker (A-F) p57kip2, (G-L) deltaC and (M-R) ath5 on the eyes of WT and ran-deficient embryos. $(\mathbf{S}, \mathbf{T})$ Expression of cell differentiation marker neuroD on the eyes of WT and ran-deficient embryos. (U-X) Expression of the cell differentiation marker $(\mathbf{U}, \mathbf{V})$ HuC/D protein and $(\mathbf{W}, \mathbf{X})$ Neurolin protein on the eyes of WT and ran-deficient embryos. (A-F) Dorsal view with anterior to the top; (G-T) lateral view with anterior to the left; (U-X) dorsal view with anterior to the top, and only the left eyes are presented to show better detail. $A L$, amacrine layer; CMZ, ciliary marginal zone; GCL, ganglion cell layer; ONL, outer nuclear layer. All images are representative of at least three repeats with $n>25$ for each repeat. 
cyclinD1 was unaffected in the early stage, but that it decreased in the later stage of ran-deficient embryos. On the contrary, p57kip2 was not expressed at either stage of ran-deficient embryos within the retinae. In addition, cell division markers, including cyclinD1 and BrdU, did not occur at the later stage, even in CMZ.

During eye development, transition of cell cycle exit to undergo differentiation is very critical. Delay in cell cycle exit will result in increased numbers of neurons or glial cells which form at the later stage. In contrast, premature cell cycle exist will result in increased numbers of neurons forming at the early stage (Ohnuma et al., 2002). Thus, premature cell cycle exit could cause the overexpression of ath5, which is the first expressed proneural gene that determines the fate of neural ganglion cells, resulting in high RGC production. However, in the absence of ath5, RGC proliferation would be affected (Willardsen et al., 2009). In this study, we observed that ath5 was not increased in ran-deficient embryos. Instead, the expression of ath5 within the retina was very low. Furthermore, we observed another proneural gene, neuroD, which contributes to determine the fate of retina precursor cells and also affects photoreceptor and amacrine interneuron differentiation. Similar to ath5 expression, results showed that neuroD expression was also severely decreased within the retinae of ran-deficient embryos.

Overexpression of ath5 and neuro $D$ induces cell cycle exit and determines subsequent cell fate (Kanekar et al., 1997; Ochocinska \& Hitchcock, 2009); however, in ran-deficient embryos, decreased cyclinD1 expression was not accompanied by the expression of ath5, neuroD, or p57kip2, and even the CMZ could not sustain proliferation (Fig. 5). Thus, we suggest that loss of Ran function inhibits neural development, disabling proliferation and differentiation of the whole progenitor pool.

Finally, HuC/D and Neurolin staining indicated that mature neurons were nearly absent from the retinae of ran-deficient embryos (Fig. 4). Taken together, our finding indicated that Ran deficiency did, indeed, result in premature cell cycle exit, but the microphthalmos phenotype was not simply caused by fewer cells. Instead, premature cell cycle exit resulted in the failure of cells to differentiate into specified retinal cells or neurons. Further, instead of staying in a static progenitor state, retinal progenitor cells under Ran deficiency lost their progenitor properties faster than their wild-type counterparts. In other words, at the late stage of eye development, Ran is required for proliferation, differentiation and also the transition from progenitor to differentiated cells in retina.

\section{Loss of Ran caused defective retinal differentiation by influencing cell division and losing other undifferentiated characteristics}

Ueno et al., (2006) treated mice with G2/M phase inhibitor 5AzC and found that the brain had many round-shaped cells which could not pass through $M$ phase since they were inhibited by $5 \mathrm{AzC}$, resulting in the accumulation of excessive cell nucleus within ventricular surface. Previous research showed that overexpression of dominant negative form of Ran within the cell results in cell cycle arrest in the G2 phase (Ren et al., 1994). Thus, in this study, we could not rule out the possibility that the defect of G2/M phase
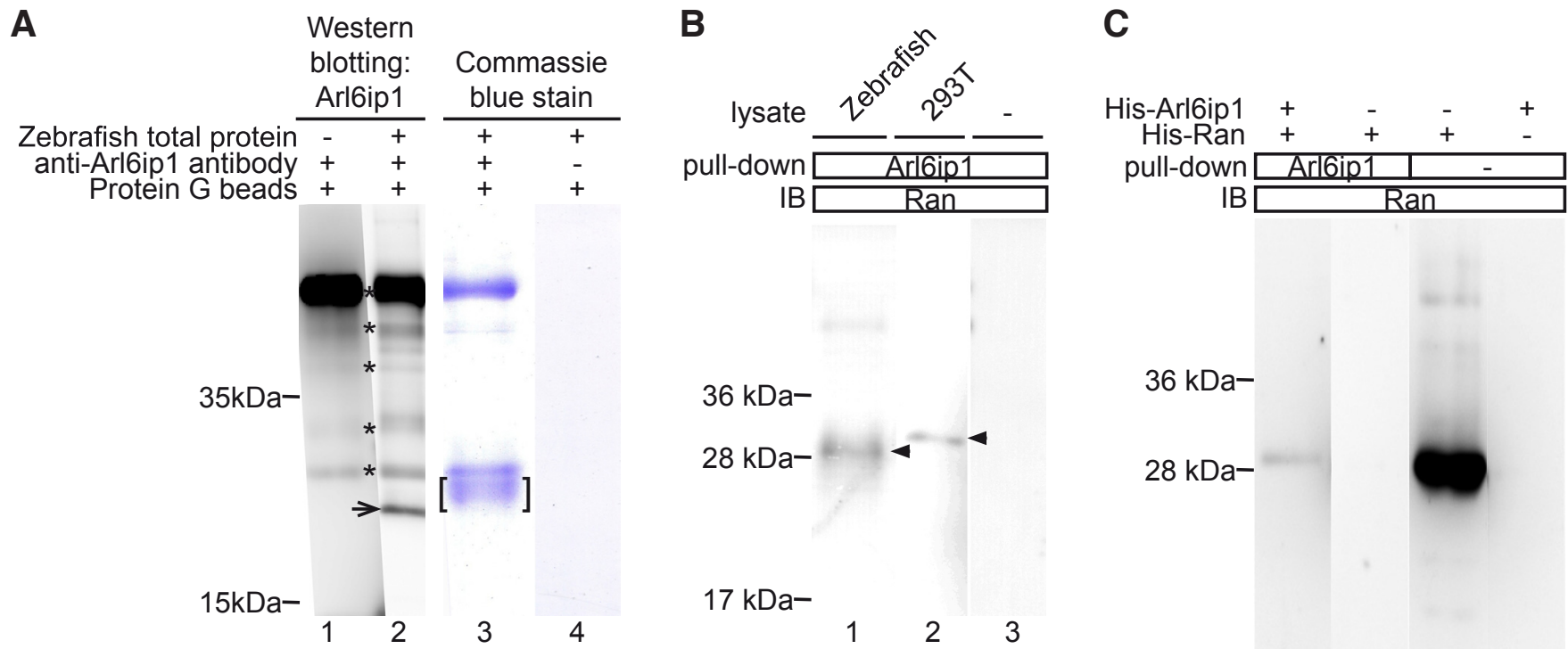

Fig. 6. Ran is a binding partner of Arl6ip1. (A) Lane 1 and lane 2: Arl6ip1 immunoprecipitation assay from whole embryonic zebrafish protein extracts. Lane 1 is the background control without adding protein extracts. Asterisks indicate that background bands exist in both lanes 1 and 2. Arrow indicates that the specific band only exists in lane 2, indicating Arl6ip1. Lane 3 is the Coomassie blue staining result of the pull-down assay. Bracket indicates that protein signals do not overlap with the molecular weights of Arl6ip1 or any background signal detected in lane 1 or 2. Lane 4 is the negative control without antibody, indicating no significant nonspecific binding of the protein $G$ beads. (B) When using the anti-Arl6ip1 antibody for pull-down and anti-Ran antibody for immunoblotting, Ran signal was detected in both whole embryonic zebrafish protein extracts and 293T whole cell lysates. Lane 1: Co-IP result of whole embryonic zebrafish protein extracts. Lane 2: Co-IP result of 293T whole cell lysates. Lane 3: Negative control without adding protein extract. Arrowheads: detected Ran signal. (C) When purified His-Arl6ip1 and His-Ran were subjected to immunoprecipitation assay, Ran signal was detected in the Arl6ip1 pull-down fraction. Lane 1: Co-IP results of His-Arl6ip1 and His-Ran. Lane 2: Negative control, in which His-Arl6ip1 was absent. Lane 3: Input control with only purified His-Arl6ip1. Lane 4: Input control with only purified His-Ran. 
transition impairs retinal development. Yet, it should be noted that we did not find HuC/D- or Neurolin-positive cells within the retinae of ran-deficient embryos, indicating that while Ran is likely to cause retina defect through influencing cell division, it may also play roles in regulating progenitor cell differentiation via mechanisms other than cell cycle regulation during retinal development.

\section{Ran associates with various binding partners to play distinct roles}

By its binding various partner, Ran is a GTPase with multiple functions. For example, Ran can associate with a centromeric protein AKAP450, and become accumulated at the centromere to facilitate cytoskeleton assembling, consequently participating in cell division (Keryer et al., 2003). Ran can also associate with RanBPM, which interacts with adhesion molecule $\mathrm{L} 1$ and axon guidance receptor plexin $A 1$, thereby affecting the growth of neurons (Cheng et al., 2005; Togashi et al., 2006). In this study, we identified Arl6ip1 as a binding partner of Ran and found that the binding between Arl6ip1 and Ran was most likely direct. Huang et al. (2009) demonstrated that Arl6ip is involved in ocular development. Arl6ip1 deficiency results in numerous developmental defects including microphthalmos. Also, the retinae of arl6ip1-deficient embryos exhibited the near absence of layered structure. Thus, we hypothesized that Ran and Arl6ip1 function in the same pathway. Indeed, not only the expression patterns of Ran and Arl6ip1 are highly similar, but the deficiency phenotypes of both are very similar, especially in the eye. Specifically, microphthalmia and defective formation of retinal layers were observed in embryos with Arl6ip1 deficiency (Huang et al., 2009). These results strongly suggest that Ran interacts with Arl6ip and that these binding partners play a role in eye development of zebrafish. However, while such findings strongly support our hypothesis, the exact downstream signaling pathway responsible for retinal development remains to be elucidated.

\section{Material and Methods}

\section{Ethics statement}

The animal protocol, which was strictly followed in this study, was reviewed and approved by the IACUC, National Taiwan University, Taiwan.

\section{Animal husbandry}

Zebrafish wild-type $A B$ strain was maintained at a temperature of $28.5^{\circ} \mathrm{C}$ with a photoperiod of $14 \mathrm{hr}$ light: $10 \mathrm{hr}$ dark. All fish were bred according to guidelines outlined in The Zebrafish Book (Westerfield, 1995).

\section{Whole-mount in situ hybridization (WISH)}

WISH was conducted following the protocol described by Huang et al., (2009), except that the chorion of embryos up to $24 \mathrm{hpf}$ was removed with Pronase $(10 \mu \mathrm{g} / \mathrm{ml})$ (Sigma). To make riboprobes, target cDNAs were subcloned into the pGEM-T easy vector (Promega). All riboprobes were labeled with digoxigenin (DIG) using DIG RNA labeling kit (Roche).

\section{Specific knockdown of ran in embryos through microinjection}

The antisense morpholino oligonucleotides (MO) that specifically inhibits the translation of ran mRNA was designed and synthesized by Gene Tools. The sequences of ran-MOs were designed as follows: ran-MO1, 5'-GAACTTGTGGCTCGTTCTCCGCCAT-3', targeting the start codon of zebrafish ran mRNA; and ran-MO2, 5'-TGTGTTGGTCTTTGTGCTGCA-3', targeting a 5'-UTR region of zebrafish ran mRNA. All ran-MOs were prepared at a stock concentration of $8.361 \mu \mathrm{g} / \mu \mathrm{l}$, stored at $-20^{\circ} \mathrm{C}$, and diluted to the desired concentration for microinjection into zebrafish embryos at the one-cell stage. Microinjection was conducted using Nanoject II (Drum- mond) microinjectors. MOs were delivered in one $2.3 \mathrm{~nL}$ pulse to the animal pole of each embryo at the one-cell stage.

\section{Histological examination}

Wild-type and ran-knockdown embryos at $72 \mathrm{hpf}$ were treated with $4 \%$ paraformaldehyde overnight at $4^{\circ} \mathrm{C}$ and embedded in paraffin. The embryos were then sectioned at $10-\mu \mathrm{m}$ intervals and subjected to standard Hematoxylin-Eosin staining.

\section{Bromodeoxyuridine (BrdU) labeling}

BrdU labeling was modified based on the procedures described by Huang et al. (2009). Dechorionated embryos were kept on ice for $15 \mathrm{~min}$ and incubated in a cold $15-\mathrm{mM} \mathrm{BrdU/1 \%}$ Dimethyl sulfoxide (DMSO) medium for $30 \mathrm{~min}$. Then, embryos were transferred to an embryonic medium at $28.5^{\circ} \mathrm{C}$ for $40 \mathrm{~min}$, fixed in $4 \%$ PFA at room temperature for $2 \mathrm{hr}$, and transferred to methanol at $-20^{\circ} \mathrm{C}$ overnight. The BrdU antibody (Abcam) was used at a dilution of 1:50. The nuclei were labeled with DAPI (Sigma) in a concentration of $45 \mathrm{ng} / \mathrm{ml}$ at room temperature for 10 to $30 \mathrm{~min}$.

\section{TUNEL assay}

TUNEL assay was conducted following the protocols described in the handbook of the DeadEnd Colormetric System (Promega).

\section{Immunofluorescent staining}

Immunofluorescent staining was performed following Huang etal. (2009). The anti-HuC/D antibody (Invitrogen) was diluted to 1:50. The anti-Neurolin antibody (Development Studies Hybridoma Bank, Product\#Zn8) was diluted to 1:200. The secondary antibody, Cy3 goat anti-mouse immunoglobulin $\mathrm{G}(\mathrm{H}+\mathrm{L})$ (Chemicon International, Cat\#AP181C), was used at a dilution of 1:200. The embryos were observed by confocal fluorescence microscope (TCS SP5, Leica).

\section{Microscopy and imaging}

The results from WISH and TUNEL assay of 24-hpf embryos were captured by a stereomicroscope (MZ FLIII, Leica) coupled with a Nikon D3 digital camera. Based on the nature of the stereomicroscope, the magnification of the captured images is arbitrary. However, the same magnification was always maintained within each WT and ran-knockdown image pair. Hematoxylin-Eosin staining and 72-hpf TUNEL assay results were captured by a compound microscope (DMR, Leica) with a 10X objective and Nikon D3 digital camera. BrdU labeling and immunostaining results were captured by a confocal microscope system (TCS SP5, Leica). Unless otherwise indicated, representative images used in this study were selected from at least three repeats and represent more than $50 \%$ of the embryos in each experimental group.

\section{Construction of expression vectors to produce recombinant Ran and Arl6ip1}

To construct expression plasmids that produced fusion recombinant proteins of His-tagged Arl6ip1 (His-Arl6ip1) and Ran (His-Ran) in Escherichia coli, we used the cDNAs of Ran and Arl6ip with Ndel and BamHI ends were constructed into the expression vector $\mathrm{pET}-15 \mathrm{~b}$. After standard IPTG induction steps, bacterial lysate containing His-Arl6ip1 was subjected to a $\mathrm{MgCl}_{2} / \mathrm{ATP} / \mathrm{KCl}$ treatment to remove the chaperonin that may be copurified. As of His-Ran, inclusion body that contained overexpressed HisRan was collected from the bacterial lysate by centrifuge. The His-tagged proteins were then purified using His GraviTrap column (GE Healthcare Life Sciences). Native protocol was used for His-Arl6ip1 while denature protocol was used for His-Ran. The extracts were subjected to in vitro immunoprecipitation assay using protein $\mathrm{G}$ magnetic beads (Millipore). Manufacturer recommended protocol was followed.

\section{Immunoprecipitation assay and Western blotting}

Whole embryo protein extracts were prepared following the protocol described by Huang et al. (2009). Protein G magnetic beads (Millipore) 
were used for immunoprecipitation. For PAGE, 10\% polyacrylamide gel, Tris/glycine-based buffer and Mini PROTEAN Tetra Cell system (Bio-Rad) were used. For transfer, PVDF membrane and Mini Trans-Blot Cell system (Bio-Rad) were used. Rabbit anti-Arl6ip1 antibody (Abcam) was used for both immunoprecipitation and Western blotting. When used on Western blotting, the antibody was diluted to 1:5000. Mouse anti-Ran antibody (Abcam) was diluted to 1:2000. Goat anti-rabbit-IgG-HRP antibody (Promega) and goat anti-mouse-IgG-HRP antibody (Promega), both diluted to 1:2000, were used as the secondary antibody.

\section{In-gel digestion and LC-MS/MS analysis}

The immunoprecipitates shown on SDS-PAGE were cut into gel pieces, and they were used to perform in-gel digestion and LC-MS/MS. The resultant MGF file was searched using the Mascot search engine (v2.2, Matrix Science) with the following conditions. 1) The protein database was set as Swiss-Prot. 2) Taxonomy was set as Danio rerio (zebrafish). 3) One trypsin missed cleavage was allowed. 4) The peptide mass tolerance was set at $\pm 0.5 \mathrm{Da}$, and the fragment mass tolerance was set at $\pm 0.5 \mathrm{Da}$. 5 ) Carbamidomethyl (Cys) was chosen as a fixed modification. 6) Oxidation (Met) and deamidation (Asn and GIn) were chosen as variable modifications.

\section{Acknowledgments}

This project was supported by the Ministry of Science and Technology, Republic of China, under grant no. NSC 98-2313-B-002-020-MY3.

\section{References}

CHENG L, LEMMON S, LEMMON V (2005). RanBPM is an L1-interacting protein that regulates L1-mediated mitogen-activated protein kinase activation. J Neurochem 94: 1102-1110.

CHOW RL, LANG RA (2001). Early eye development in vertebrates. Annu Rev Cell Dev Biol 17: 255-296.

CHUANG JC, RAYMOND PA (2001). Zebrafish genes rx1 and rx2 help define the region of forebrain that gives rise to retina. Dev Biol 231: 13-30.

CICIARELLO M, MANGIACASALE R, LAVIA P (2007). Spatial control of mitosis by the GTPase Ran. Cell Mol Life Sci 64: 1891-1914

DASSO M (2001). Running on Ran: nuclear transport and the mitotic spindle. Cell 104: $321-324$

DENG L, SHANG Y, GUO S, LIU C, ZHOU L, SUN Y, NIE Y, FAN D, LU Y, GUO X (2014). Ran GTPase protein promotes metastasis and invasion in pancreatic cancer by deregulating the expression of AR and CXCR4. Cancer Biol Ther 15: 1087-1093.

GORLICHD, KUTAYU (1999). Transport between the cell nucleus and the cytoplasm. Annu Rev Cell Dev Biol 15: 607-660.

HEISENBERG CP, BRENNAN C, WILSON SW (1999). Zebrafish aussicht mutant embryos exhibit widespread overexpression of ace (fgf8) and coincident defects in CNS development. Development 126: 2129-2140.

HETZER M, GRUSS OJ, MATTAJ IW (2002). The Ran GTPase as a marker of chromosome position in spindle formation and nuclear envelope assembly. Nat Cell Biol 4: E177-184.

HUANG HY, DAI ES, LIU JT, TU CT, YANG TC, TSAI HJ (2009). The embryonic expression patterns and the knockdown phenotypes of zebrafish ADP-ribosylation factor-like 6 interacting protein gene. Dev Dyn 238: 232-240.

KANEKAR S, PERRON M, DORSKY R, HARRIS WA, JAN LY, JAN YN, VETTER ML (1997). Xath5 participates in a network of bHLH genes in the developing Xenopus retina. Neuron 19: 981-994.

KIM CH, UESHIMA E, MURAOKA O, TANAKA H, YEO SY, HUH TL, MIKI N (1996). Zebrafish elav/HuC homologue as a very early neuronal marker. Neurosci Lett 216: 109-112.

KERYER G, DI FIORE B, CELATI C, LECHTRECK KF, MOGENSEN M, DELOUVEE A, LAVIA P, BORNENS M, TASSIN AM (2003). Part of Ran is associated with AKAP450 at the centrosome: involvement in microtubule-organizing activity. $\mathrm{Mo}$ Biol Cell 14: 4260-4271

KRAUSS S, JOHANSEN T, KORZH V, FJOSE A (1991). Expression of the zebrafish paired box gene pax[zf-b] during early neurogenesis. Development 113: 1193-1206.

LIAO J, HE J, GONG Z (1997). An abundant zebrafish cDNA clone encodes a ras-like protein which is expressed ubiquitously. DNA Seq 7: 313-317.

MELCHIOR F, GUAN T, PASCHALB, GERACE L (1995). Biochemical and structural analysis of nuclear protein import. Cold Spring Harb Symp Quant Biol60: 707-716.

OCHOCINSKA MJ, HITCHCOCK PF (2009). NeuroD regulates proliferation of photoreceptor progenitors in the retina of the zebrafish. Mech Dev 126: 128-141.

OHNUMAS, HOPPERS, WANG KC, PHILPOTTA, HARRISWA(2002). Co-ordinating retinal histogenesis: early cell cycle exit enhances early cell fate determination in the Xenopus retina. Development 129: 2435-2446.

PINES J (1995). Cyclins and cyclin-dependent kinases: a biochemical view. Biochem J 308: 697-711.

REN M, COUTAVAS E, D'EUSTACHIO P, RUSH MG (1994). Effects of mutant Ran TC4 proteins on cell cycle progression. Mol Cell Biol 14: 4216-4224.

SAZER S (1996). The search for the primary function of the Ran GTPase continues. Trends Cell Biol 6: 81-85.

SAZER S, DASSO M (2000). The ran decathlon: multiple roles of Ran. J Cell Sci 113: $1111-1118$.

SHKUMATAVA A, NEUMANN CJ (2005). Shh directs cell-cycle exit by activating p57Kip2 in the zebrafish retina. EMBO Rep 6: 563-569.

SMITHERS L, HADDON C, JIANG YJ, LEWIS J (2000). Sequence and embryonic expression of deltaC in the zebrafish. Mech Dev 90: 119-123.

TOGASHI H, SCHMIDT EF, STRITTMATTER SM (2006). RanBPM contributes to Semaphorin3A signaling through plexin-A receptors. J Neurosci 26: 4961-4969.

UENO M, KATAYAMAK, YAMAUCHI H, NAKAYAMA H, DOI K (2006). Cell cycle and cell death regulation of neural progenitor cells in the 5-azacytidine (5AzC)-treated developing fetal brain. Exp Neurol 198: 154-166.

WARD ME, TAUBESA, CHEN R, MILLER BL, SEPHTON CF, GELFAND JM, MINAM S, BOSCARDIN J, MARTENS LH, SEELEY WW, YU G, HERZ J, FILIANO AJ, ARRANT AE, ROBERSON ED, KRAFT TW, FARESE RV JR, GREEN A, GAN $L$ (2014). Early retinal neurodegeneration and impaired Ran-mediated nuclear import of TDP-43 in progranulin-deficient FTLD. J Exp Med 211: 1937-1945.

WESTERFIELD M (1995). A Guide for the Laboratory Use of Zefrafish (Danio rerio), OR: University of Oregon Press, Eugene.

WILLARDSEN MI, SULIA, PANY, MARSH-ARMSTRONG N, CHIEN CB, EL-HODIR $\mathrm{H}$, BROWN NL, MOORE KB, VETTER ML (2009). Temporal regulation of Ath5 gene expression during eye development. Dev Biol 326: 471-481.

ZAGHLOUL NA, MOODY SA (2007). Alterations of rx1 and pax6 expression levels at neural plate stages differentially affect the production of retinal cell types and maintenance of retinal stem cell qualities. Dev Biol 306: 222-240. 


\section{Further Related Reading, published previously in the Int. J. Dev. Biol.}

Pregnancy-specific glycoproteins: complex gene families regulating maternal-fetal interactions

Tom Moore and Gabriela S. Dveksler

Int. J. Dev. Biol. (2014) 58: 273-280

http://dx.doi.org/10.1387/ijdb.130329gd

\section{AP-1 C-Jun/FosB $^{-}$mediates xFoxD5b expression in Xenopus early developmental neurogenesis}

Jaeho Yoon, Jung-Ho Kim, Ok-Joo Lee, Sung-Young Lee, Seung-Hwan Lee, Jae-Bong Park, Jae-Yong Lee, Sung-Chan Kim and Jaebong Kim Int. J. Dev. Biol. (2013) 57: 865-872

http://dx.doi.org/10.1387/ijdb.130163jk

\section{Regulation of Merkel cell development by Pax6}

Ida Parisi and J. Martin Collinson

Int. J. Dev. Biol. (2012) 56: 341-350

http://dx.doi.org/10.1387/ijdb.113406ip

Signal transduction in vasculogenesis and developmental angiogenesis

Sunita Patel-Hett and Patricia A. D'Amore

Int. J. Dev. Biol. (2011) 55: 353-363

http://dx.doi.org/10.1387/ijdb.103213sp

Zebrafish grainyhead-like1 is a common marker of different non-keratinocyte epidermal cell lineages, which segregate from each other in a Foxi3-dependent manner Martina Jänicke, Björn Renisch and Matthias Hammerschmidt

Int. J. Dev. Biol. (2010) 54: 837-850

http://dx.doi.org/10.1387/ijdb.092877mj

Molecular tools, classic questions - an interview with Clifford Tabin Michael K. Richardson

Int. J. Dev. Biol. (2009) 53: 725-731

http://dx.doi.org/10.1387/ijdb.072575mr

Pattern formation in the Drosophila eye disc Jean-Yves Roignant and Jessica E. Treisman

Int. J. Dev. Biol. (2009) 53: 795-804

http://dx.doi.org/10.1387/ijdb.072483jr

5 yr ISI Impact Factor $(2013)=2.879$

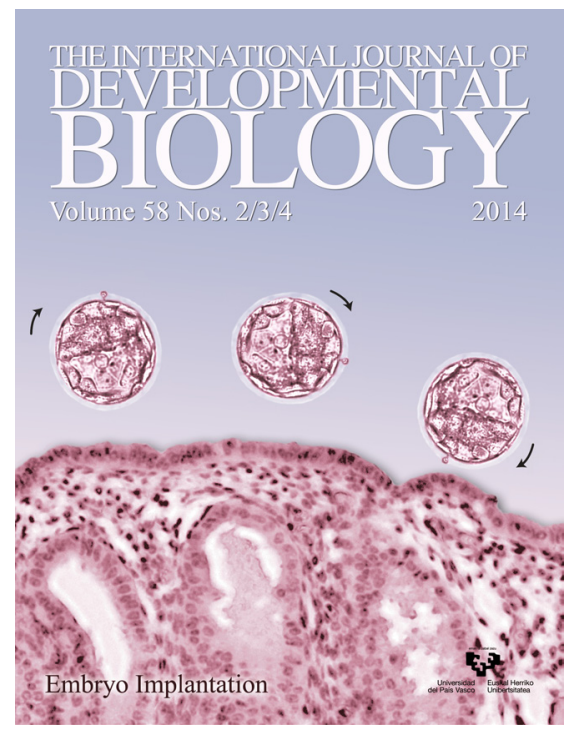

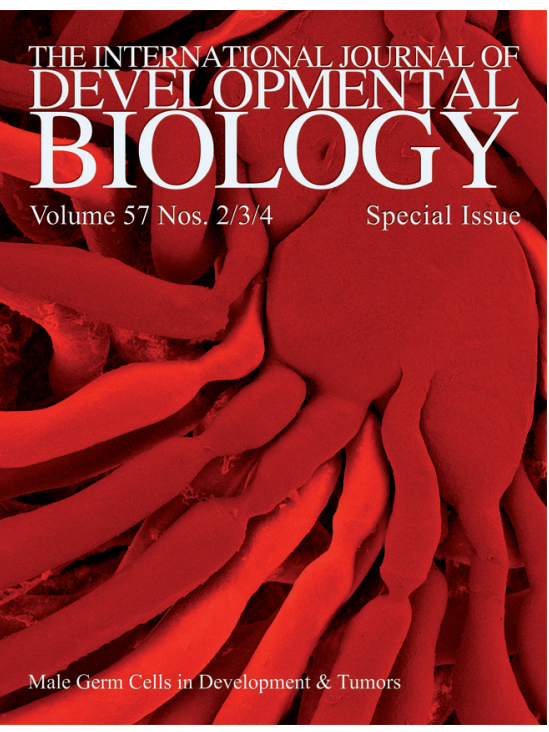
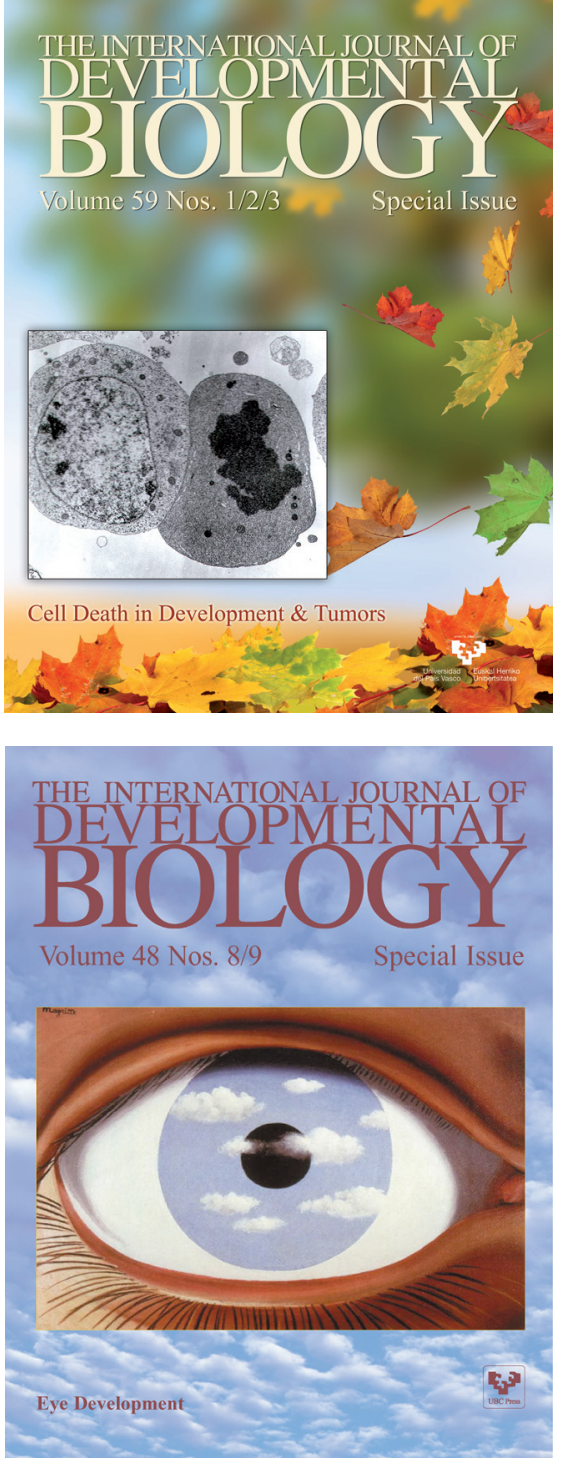